\title{
Neph1 and nephrin interaction in the slit diaphragm is an important determinant of glomerular permeability
}

\author{
Gang Liu, ${ }^{1}$ Beenu Kaw, ${ }^{2}$ Jayson Kurfis, ${ }^{1}$ Syed Rahmanuddin, ${ }^{1}$ Yashpal S. Kanwar, ${ }^{3}$ \\ and Sumant S. Chugh ${ }^{1}$ \\ ${ }^{1}$ Division of Nephrology, Department of Medicine, Feinberg School of Medicine, Northwestern University, \\ Chicago, Illinois, USA \\ ${ }^{2}$ Department of Medicine, Illinois Masonic Medical Center, Chicago, Illinois, USA \\ ${ }^{3}$ Department of Pathology, Feinberg School of Medicine, Northwestern University, Chicago, Illinois, USA
}

\begin{abstract}
Neph1-deficient mice develop nephrotic syndrome at birth, indicating the importance of this protein in the development of a normal glomerular filtration barrier. While the precise subcellular localization of Neph1 remains unknown, its relationship with other components of the glomerular filtration barrier is of great interest in this field. In this paper, we localize the expression of Neph1 to the glomerular slit diaphragm by immunogold electron microscopy in rodents and describe its direct interaction with two other components of the slit diaphragm, nephrin and ZO-1. Both native and recombinant Neph1 associate with each other as dimers and multimers and interact with nephrin via their extracellular segments. Disruption of the Neph1-nephrin interaction in vivo by injecting combinations of individual subnephritogenic doses of anti-Neph1 and anti-nephrin results in complement- and leukocyte-independent proteinuria with preserved foot processes. This disruption modestly reduces Neph 1 and nephrin protein expression in podocytes and dramatically reduces ZO-1 protein expression via the interaction of ZO-1 PDZ domains with the cytoplasmic tail of Neph1, independent of changes in mRNA expression of all three genes. The interaction between nephrin and Neph1 is specific and not shared by either protein with P-cadherin, another integral slit diaphragm protein. The interaction between nephrin and Neph1 therefore appears to be an important determinant of glomerular permeability.
\end{abstract}

J. Clin. Invest. 112:209-221 (2003). doi:10.1172/JCI200318242.

\section{Introduction}

A large number of genes with relevance to the biology of the podocyte and slit diaphragm have recently been characterized. Some encode transcriptional factors and are thought to regulate the expression of podocyte proteins (1-3), but the vast majority encode for structural components of the podocyte foot process or the slit diaphragm. Whereas the localization of ZO-1 (4) and the mAb 5-1-6 antigen (5) to the cytoplasmic and extracellular aspects, respectively, of the slit diaphragm were in themselves stellar observations, the discovery of nephrin (6) opened the field for the identification of other novel proteins. The rapid discovery of CD2 AP (7), podocin (8), $\alpha$-actinin 4 (9), P-cadherin (10), and FAT (11) as components of the podocyte foot process/slit diaphragm complex, the recent identification of filtrin (12), and the recharacterization of $\mathrm{mAb}$

Received for publication February 28, 2003, and accepted in revised form May 6, 2003.

Address correspondence to: Sumant S. Chugh, Division of Nephrology, Department of Medicine, Feinberg School of Medicine, Northwestern University, 320 East Superior Avenue, Searle 10-475, Chicago, Illinois 60611, USA.

Phone: (312) 503-3072; Fax: (312) 503-0622

E-mail: s-chugh@northwestern.edu.

Conflict of interest: The authors have declared that no conflict of interest exists.

Nonstandard abbreviations used: rapid amplification of cDNA ends (RACE); APA, aminopeptidase A.
5-1-6 as an anti-nephrin $\mathrm{Ab}(13)$ are just some of the important developments over the past 4 years.

This study addresses the characterization of Neph1, a gene recently mutated in mouse embryonic stem cells using a high-throughput mutagenesis method (14). Mutant mice generated from these stem cells develop nephrotic syndrome at birth, underscoring the importance of Neph1 in the development of a normal glomerular permeability barrier. Electron microscopy of the kidneys from the mutant mice revealed the absence of normal foot process development, and Lac- $Z$ staining of these sections revealed the presence of Neph1- $\beta$-geo fusion transcripts in several cell types, including podocytes. A single 9-kb transcript was noted by Northern blot of several organs, with high expression in the kidney. Whereas the human Neph1 cDNA sequence published by Donoviel et al. (14) encoded for five Ig-like domains and a transmembrane domain, the mouse counterpart had only four Ig-like domains and lacked a transmembrane domain, suggesting that it was a partial clone. A more recent paper (15) included both fulllength mouse and human sequences, and described the interaction of Neph1 with podocin. Whereas the expression of Neph1 in the podocyte is not in doubt, its localization in the slit diaphragm has been inferred from the nephrotic phenotype of Neph1 $1^{-/-}$mice and its protein-protein interactions (16). 
In this paper, we provide evidence for the subcellular localization of Neph1 in the glomerular slit diaphragm by immunogold electron microscopy. We present evidence that Neph1 interacts with nephrin in vitro and in vivo, and that this heterologous protein-protein interaction is an important factor in maintaining the normal permeability characteristics of the slit diaphragm. We also show the association of PDZ domains of ZO-1 with the cytoplasmic tail of Neph1 and characterize a novel model of the functional properties of the slit diaphragm.

\section{Methods}

Cloning of mouse Neph1 cDNA. Using primers designed from the published incomplete clone, we identified a full-length Neph 1 cDNA by $3^{\prime}$ rapid amplification of cDNA ends (RACE) and RT-PCR in the following manner. Total RNA was extracted from 4-week-old male C57BL/6 mouse kidney using TRIzol reagent (Invitrogen Corp., Carlsbad, California, USA), and cDNA templates were generated by reverse transcription using SuperScript II (Invitrogen Corp.). Using primers at either end of the published sequence, PCR fragments were cloned into PCRII (Invitrogen Corp.), sequenced, and compared with the published sequence. Using primers K7 (5'-GCAGGAGGGGGCTGTGACTAGCACGGAGC-3') and K8 (nested primer 5'-GGGGGCTGTGACTAGCACGGAGCTGC-3') from this sequence, $3^{\prime}$ RACE was performed using the GeneRacer kit (Invitrogen Corp.) per the manufacturer's instructions. A 2,673-bp RACE product was cloned into PCRII, and both sense and antisense strands were sequenced up to the polyA tail. Upon combining both sets of clones, a stop codon was noted 2,367 bp downstream of the open reading frame. Using primers overlapping the start and stop codons, several full-length cDNA clones were generated, sequenced, and compared for sequence identity. Protein translation, domain and motif analysis, and sequence comparisons were done using software on the BLAST (17), Scansite (18), and ExPASy (19) websites. Signal peptide prediction was done using SignalP version 1.1 software on the CBS World Wide Web Prediction server (20) and transmembrane domain prediction was done on the "DAS" Transmembrane Prediction server (21).

Since several experiments in rats were anticipated, we also cloned, sequenced, and assembled a partial rat Neph1 cDNA sequence using two primer pairs from the mouse sequence (K281, 5' -GTACAGTGGACCAAGGACGGGCTGG$3^{\prime}$ and K283, 5' -CTGCATGCGCTGCTGGAATCGCTGGCC$3^{\prime}$; K282, 5' -GTCTGATGACGCTTCCTATGAGTGCCAGG-3' and K284, 5'-CTCATAGTTCTCGTAGGACAGCTGGCTGG$\left.3^{\prime}\right)$ and rat kidney cDNA. The rat cDNA sequence and its predicted protein sequence were used primarily to design real-time PCR primers and peptides for $A b$ generation, respectively.

Raising polyclonal $A b$ 's. The extracellular segments of several mouse podocyte proteins were analyzed by hydrophobicity plots using Kyte-Doolittle curves on the ProtScale web site (22); the most hydrophilic portions specific to the protein were selected for peptide synthesis. Peptide sequences that were chosen were homologous or nearly homologous (maximum of one AA difference) with corresponding rat sequences. The Neph1 peptide sequences that were synthesized had perfect homology with both mouse and rat Neph1 proteins. Prior to immunization, $15 \mathrm{ml}$ of preimmune serum was obtained from each rabbit. Two KLH-conjugated peptides (of $15 \mathrm{AAs}$ each) for each protein were used to immunize two rabbits each to raise polyclonal Ab's. Initial immunization was done with complete Freund's adjuvant, whereas boosters at days 20, 40, and 60 were given with incomplete Freund's adjuvant. ELISA titers for antigen-specific reactivity were checked after the first bleed, at day 70 , using peptide coated wells. The following Ab's were raised: anti- $\alpha$-dystroglycan (PNQRPELKNHIDRVD, STTTTTRRPTKKPRTP), anti- $\beta$-dystroglycan (RRIADENGKPRPAFS, TEVPDRDPEKSSEDD), anti-nephrin (DLQDPRYTEHKYHQG, FPRYSLEGDSAKGEF), anti-aminopeptidase A (YVQPNQKETAEYAA, YTLEQYQKTSLAQEK), and anti-Neph1 (EDAHESRYETNVDYS, RSMNEAIPNGKETSI). These Ab's were raised by Genemed Synthesis Inc. (South San Francisco, California, USA) on a fee-for-service basis.

Rabbit anti-ZO-1 and rat anti-P-cadherin with reactivity against the extracellular domain of $\mathrm{P}$-cadherin (10) were purchased from Zymed Laboratories Inc. (South San Francisco, California, USA). Other Ab's purchased included mouse anti-rat CD45 (Cedarlane Laboratories Ltd., Hornby, Ontario, Canada), goat anti-mouse CD2 AP (Santa Cruz Biotechnology Inc., Santa Cruz, California, USA), goat anti-rabbit IgG coupled with 10-nm gold particles (Electron Microscopy Services, Fort Washington, Pennsylvania, USA), rabbit anti-goat IgG coupled with 15 -nm gold particles (Electron Microscopy Services), and goat anti-rat C3 (ICN Biomedical Inc., Aurora, Ohio, USA). Secondary Ab's for immunofluorescence and Western blotting were purchased from Sigma-Aldrich (St. Louis, Missouri, USA). Rabbit anti-rat albumin Ab was obtained from Research Diagnostics Inc. (Flanders, New Jersey, USA).

General laboratory reagents were obtained from Sigma-Aldrich. Reagents for electron microscopy were purchased from Electron Microscopy Services. Laboratory animals were purchased from Harlan Sprague Dawley Inc. (Indianapolis, Indiana, USA).

Animal studies. All animal studies were approved by the Animal Care and Use Committee of Northwestern University. In an attempt to study an in vivo association between various podocyte proteins as measured by the induction of proteinuria, the following groups of experiments were performed in male Sprague Dawley rats and female CD1 mice (four animals/group). Baseline 18-hour urinary protein excretion was performed for each animal used in the study. The choice of relatively young rats (approximately $100 \mathrm{~g}$ ) whenever possible in these experiments is governed by the fact that they have 
very little measurable baseline proteinuria. For the induction of proteinuria, all $\mathrm{Ab}$ 's or antisera were heattreated at $56^{\circ} \mathrm{C}$ for 30 minutes to inactivate complement prior to intravenous injection into rats or mice.

To study in vivo interactions between Neph1 and nephrin or P-cadherin, dose-response studies with anti-Neph1, anti-nephrin, and anti-P-cadherin were first performed by injecting rats via the tail vein with up to $500 \mu \mathrm{l}$ of antisera (anti-nephrin, anti-Neph1, or preimmune serum) or up to $100 \mu \mathrm{g}$ of anti-P-cadherin. The goal of this study was to define the optimum subnephritogenic dose of these Ab's beyond which a mild increase in slit diaphragm permeability would occur. The rats were placed in metabolic cages with access to water but no food for an 18-hour urine collection after injection. Proteinuria was measured using a protein assay kit from Bio-Rad Laboratories Inc. (Hercules, California, USA) based on the Bradford method; readings were multiplied by 1.33 to adjust for a 24-hour collection. Wherever proteinuria was noted, urine samples were appropriately diluted in PBS, resolved by SDS PAGE, and stained with GelCode Blue (Pierce Biotechnology Inc., Rockford, Illinois, USA). Samples were also processed for Western blot with an anti-rat albumin $\mathrm{Ab}$. To test for an in vivo association between two proteins, the highest subnephritogenic dose of one of two Ab's or a combination of $50 \%$ of that dose of both Ab's was intravenously injected into three different groups of animals. In the anti-Neph1 and anti-nephrin study, two groups of rats were injected with $300 \mu$ of either antiNeph1 or anti-nephrin, while a third group received a combination of $150 \mu \mathrm{l}$ of each $\mathrm{Ab}$. A fourth group (control) received $300 \mu \mathrm{l}$ of preimmune serum from the same rabbits. For the anti-Neph1 and anti-P-cadherin study, rats received either $300 \mu$ of anti-Neph1, $75 \mu \mathrm{g}$ of anti-P-cadherin, a combination of $50 \%$ of these doses, or $300 \mu \mathrm{l}$ of preimmune serum. We also intravenously injected increasing doses up to $250 \mu \mathrm{l}$ of preimmune serum, anti-nephrin, anti- $\alpha$-dystroglycan, anti- $\beta$-dystroglycan, and anti-aminopeptidase A (anti-APA) separately into 8- to 12-week-old female CD1 mice, and analyzed the collected urine for proteinuria. Since rat glomeruli can be isolated by sieving with over $90 \%$ purity, most of the in vivo studies that required measurement of glomerular protein and mRNA expression were conducted in rats.

In all rodent studies, kidneys were removed after euthanasia and appropriate portions were used for fresh glomerular isolation for protein or total RNA extraction, or preserved for electron microscopy (with $2.5 \%$ glutaraldehyde), light microscopy (with 3.7\% formaldehyde), or frozen sections (snap-frozen and preserved in 2-methylbutane).

Expression of FLAG-tagged proteins. To study their relative roles in protein-protein interactions, cDNA fragments corresponding to both extracellular and intracellular segments of Neph 1 were cloned separately into PCAL-nFLAG (Stratagene, La Jolla, California, USA) in the following manner. A 1,424-bp fragment of mouse Neph1 cDNA corresponding to the extracellular segment was cloned into pCAL-n-FLAG between the EcoRI and HindIII sites using primers K227 (GCGCGCGAATTCCCCGGGACTCAGACTCGCTTCAGCC) and K230 (GCGCGCAAGCTTCTATAACACCTCTCGCTCTTCCAGCTGG). A 717-bp fragment corresponding to the intracellular segment was cloned between the same restriction enzyme sites using primers K229 (GCGCGCGAATTCCTCTACCGACGTCGCAAAGGCAGTC) and K233 (GCGCGCAAGCTTTCTACACATGAGTCTGCATGCGCTGCTGG). A segment of mouse ZO-1 containing the three PDZ domains was also cloned between the BamHI and HindIII sites of pCAL-n-FLAG using primers K238 (GCTATAGGATCCAAGATGTCCGCCAGGGCCGCGG) and K239 (GCGCTTAAGCTTTGCGGCGATAAACGTCCTTCTTC) and a cDNA template derived from mouse kidney total RNA by reverse transcription.

After verifying the accuracy of the plasmids by sequencing, protein expression was induced with isopropyl- $\beta$-D-thiogalactoside (IPTG) in BL21-Gold (DE3) E. coli (Stratagene) according to the product manual. Protein expression was verified by resolving preinduced and induced cultures by SDS PAGE under reducing and nonreducing conditions and staining with GelCode Blue, as well as transferring the proteins to nitrocellulose for Western blot with anti-FLAG (all expressions) or anti-Neph1 (extracellular segment only). The purified recombinant proteins were column-purified, dialyzed against PBS, and stored at $-80^{\circ} \mathrm{C}$. To generate non-FLAG-tagged recombinant protein, the FLAG domain and the calmodulin-binding peptide were cleaved from the recombinant protein using enterokinase as recommended in the product manual for PCALn-FLAG. Non-FLAG-tagged and FLAG-tagged recombinant proteins were used in competition experiments.

Western blot and coimmunoprecipitation studies. For glomerular studies involving the characterization of rat/mouse Neph1 and for coimmunoprecipitation studies, a standard protocol described previously was used (23). Autoradiographs were scanned into Adobe Photoshop 5.0, and band densitometry was measured using Gel-Pro Analyzer 3.1 software (Media Cybernetics Inc., Silver Spring, Maryland, USA).

For studies involving the injection of anti-Neph1, anti-nephrin, or both into rats, glomerular proteins were extracted by a different protocol (24) that also allowed for the concomitant extraction of ZO-1, which is resistant to detergent extraction by regular nonionic detergents like $n$-octyl- $\beta$-D-glucopyranoside or Triton X-100. Glomeruli were isolated by differential sieving and washed with PBS, protease inhibitors were added, and $100 \mu$ of the pellet was solubilized by vortexing in $200 \mu \mathrm{l}$ of SDS PAGE sample buffer without bromophenol blue. Insoluble material was removed by centrifugation at $15,000 \mathrm{~g}$ for 10 minutes. The protein concentration was measured by the BCA protein assay (Pierce Biotechnology Inc.) and $30 \mu \mathrm{g}$ of protein was loaded into each lane and resolved by 
a

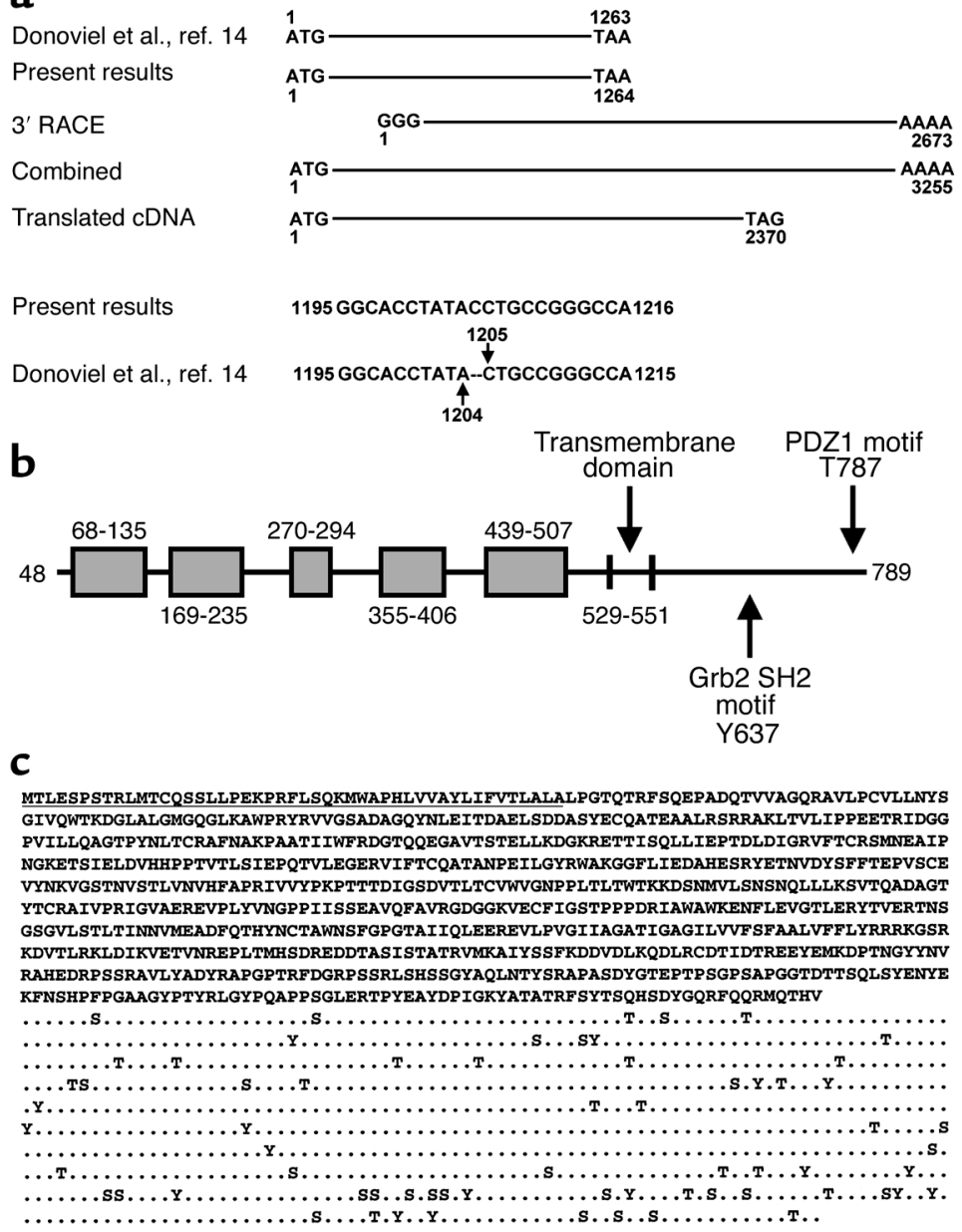

Figure 1

Cloning and characterization of mouse Neph1. (a) The missing base pair after 1,204 in the original published sequence, and the assembly of full-length mouse cDNA by 3'RACE. (b) A representation of mouse Neph1 protein structure, including five Iglike domains in the extracellular segment. (c) The full-length Neph1 protein prior to signal peptide (underlined) cleavage, along with potential phosphorylation sites.

immunoprecipitates with $0.1 \mathrm{M} 2$-mercaptoethanol and $0.1 \% \mathrm{SDS}$, followed by overnight incubation at $37^{\circ} \mathrm{C}$ with either PNGase F or control. The proteins were then resolved by reducing SDS PAGE and Western blot with anti-Neph1 Ab.

Affinity absorption of anti-Neph1 Ab with Neph1 peptides and recombinant extracellular segment of Neph1. We coupled $3 \mathrm{mg}$ of each of the two peptides used to generate the anti-Neph $1 \mathrm{Ab}$ with $1 \mathrm{ml}$ of CNBr-activated Sepharose 4B (Amersham Pharmacia Biotech, Uppsala, Sweden) according to the manufacturer's instructions to make an affinity column that was used to absorb out reactivity to these peptides from the anti-Neph1 Ab. Depletion of anti-Neph1 activity was assessed by Western blot and immunofluorescence. A similar strategy was adopted for using nephrin peptides to absorb out reactivity to nephrin from the anti-nephrin $\mathrm{Ab}$. A second affinity column for mouse Neph 1 was made with $3 \mathrm{mg}$ of the recombinant extracellular segment of Neph1 after the FLAG tag and the calmodulin-binding peptide tag were removed using enterokinase, and was tested in a similar manner to the first affinity column.

Real-time PCR for Neph1, nephrin, and ZO-1. Real-time PCR was used to study rat glomerular mRNA expression of nephrin, Neph1, and ZO-1 in the groups injected with anti-nephrin, anti-Neph1, or a combination of the two as described above. Glomeruli were isolated from each rat by sieving, and $2 \mu \mathrm{g}$ of total RNA was used to generate cDNA by reverse transcription. Three cDNA templates were generated from each rat so that every animal was assayed in

SDS PAGE. Following transfer onto nitrocellulose membranes, Western blot studies were conducted with anti-Neph1, anti-nephrin, and anti-ZO-1.

For coimmunoprecipitation studies involving FLAGtagged recombinant proteins and native glomerular protein extracts, the amount of FLAG-tagged protein to be added to the glomerular extract was determined in the following manner. Thirty micrograms of the glomerular extract was resolved by SDS PAGE along with different concentrations of recombinant protein and stained with GelCode Blue (Pierce Biotechnology Inc.). We then determined the recombinant protein band that appeared to be roughly equivalent in intensity to corresponding bands at the same molecular weight in the membrane extract. The protein concentration of the selected band determined the amount of the FLAGtagged protein added to the glomerular extract.

Deglycosylation studies. To study whether native mouse Neph1 from glomerular extracts was actually glycosylated as predicted, we denatured anti-Neph1 triplicate. The following primers and TaqMan probes for real-time PCR were designed using Primer Express version 2.0 software (Applied Biosystems, Foster City, California, USA). Rat nephrin: H7 forward primer $5^{\prime}$ TTCAGCAAGGAGACCTTCAAGAA-3', H8 reverse primer 5'-GCCCCTCAATCCACAGCTT-3', probe 6FAM-TCACTCACCTTGAATGTGA-MGB; rat ZO-1: H13 forward primer 5'-GAGCTACGCTTGCCACACTGT-3', H14 reverse primer 5'-TCGGATCTCCAGGAAGACACTT-3', probe 6FAM-ACCCTAAAACTTGGCAAAA-MGB; rat Neph1: H3 forward primer $5^{\prime}$-TTGCTGCCTTAGTGTTCTTTCTCTAC$3^{\prime}, \mathrm{H} 4$ reverse primer $5^{\prime}$-CCTCAACGTCACATCCTTTCG-3', probe 6FAM-ACGTCGCAAAGGCAGT-MGB.

Forward and reverse primers were designed in adjacent exons to avoid false amplification by possible contaminating genomic DNA. All real-time PCR products from individual primer pairs were sequenced to ensure the accuracy of the PCR reaction. Control 18S RNA probe (VIC-MGB) and corresponding primers were purchased from Applied Biosystems. Real-time PCR was 
performed in a 96-well format on the ABI Prism 7000 (Applied Biosystems) in multiplex mode (i.e., study primers/probe plus $18 \mathrm{~S}$ control primers/ probe) using preset cycling parameters specified by the manufacturer. Appropriate nontemplate controls were included in each experiment. Prior to the run, threshold settings were set at 0.2. After the run was concluded, the data was reanalyzed to obtain $\mathrm{Ct}$ values with a threshold setting of 0.75 in $\Delta \mathrm{Rn}$ versus Cycle mode for all groups. $\Delta$ Ct values for each individual reading were calculated by subtracting the corresponding $18 \mathrm{~S} \mathrm{Ct}$ values from each study group reading, mean $\pm \mathrm{SE}$ was calculated for each group, and differences between combination and individual $\mathrm{Ab}$ groups were analyzed by Student $t$ test. In addition, we subtracted the $\Delta \mathrm{Ct}$ of the individual $\mathrm{Ab}$ injected groups from that of the combination injected group to obtain the $\Delta \Delta \mathrm{Ct}$ and calculated the difference in mRNA expression between these groups using the formula $2^{-\Delta \Delta C t}$ (see Applied Biosystems ABI 7000 user bulletin no. 2 for sequence detection at http://docs.appliedbiosystems.com/search. taf). A fourfold difference in mRNA expression, equivalent to a mean difference of two cycles by real- time PCR, was used as a criterion to define a significant difference between the groups.

Imaging studies. For immunofluorescence studies, a standard protocol recently described in detail was used (23). The positive control for complement staining with anti-rat $\mathrm{C} 3 \mathrm{Ab}$ was day 5 kidney of a rat injected with sheep anti-rat Fx1A. Positive control for leukocyte staining with anti-CD45 was kidney from a rat injected with whole sheep anti-rat nephrotoxic serum 24 hours prior to euthanasia.

For immunogold electron microscopy of perfused rat kidney and for transmission electron microscopy, standard protocols described elsewhere were used $(25,26)$. The positive control for the procedure of immunogold localization of Neph1 was staining for CD2 AP in rat glomeruli. In addition, other rats were injected separately with $150 \mu \mathrm{l}$ of either anti-Neph 1 or anti-nephrin 1 hour prior to perfusion fixation, followed by staining with $10 \mathrm{~nm}$ gold-coupled goat anti-rabbit IgG to assess for binding of injected Ab's to the slit diaphragm.

Statistical analysis to study the difference in protein expression between different experimental groups was done using the unpaired Student $t$ test in Microsoft Excel 2000. A $P$ value of $<0.05$ was taken as significant.
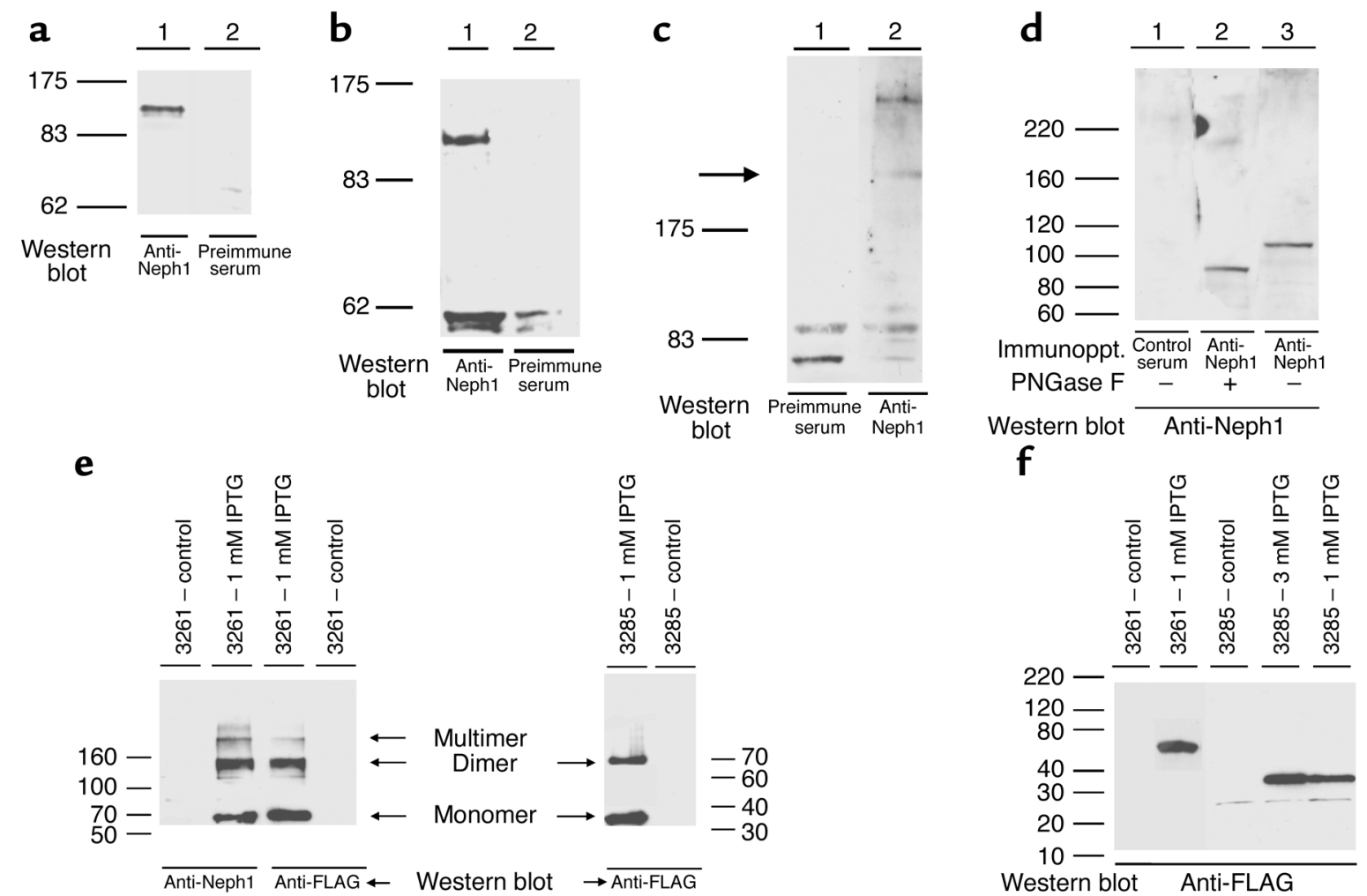

Figure 2

Characterization of native and recombinant mouse Neph 1 by Western blot. ( $\mathbf{a}$ and $\mathbf{b}$ ) A distinct band is seen at approximately $110 \mathrm{kDa}$ in rat and mouse glomerular extracts, respectively, under reducing conditions. (c) There is a band at approximately $220 \mathrm{kDa}$ (arrow) and a higher band in mouse glomerular extracts under nonreducing conditions, suggesting dimer and multimer formation. (d) Migration of antiNeph1 immunoprecipitates (Immunoppt.) from mouse glomerular extracts at approximately $90 \mathrm{kDa}$ following deglycosylation with PNGase F. (e and f) Composite Western blots of the extracellular segment (clone 3,261) and intracellular segment (clone 3,285) expressed in E. coli by IPTG induction, run under nonreducing $(\mathbf{e})$ and reducing $(\mathbf{f})$ conditions, also demonstrating dimer formation under nonreducing conditions. IPTG, isopropyl- $\beta$-D-thiogalactoside. 

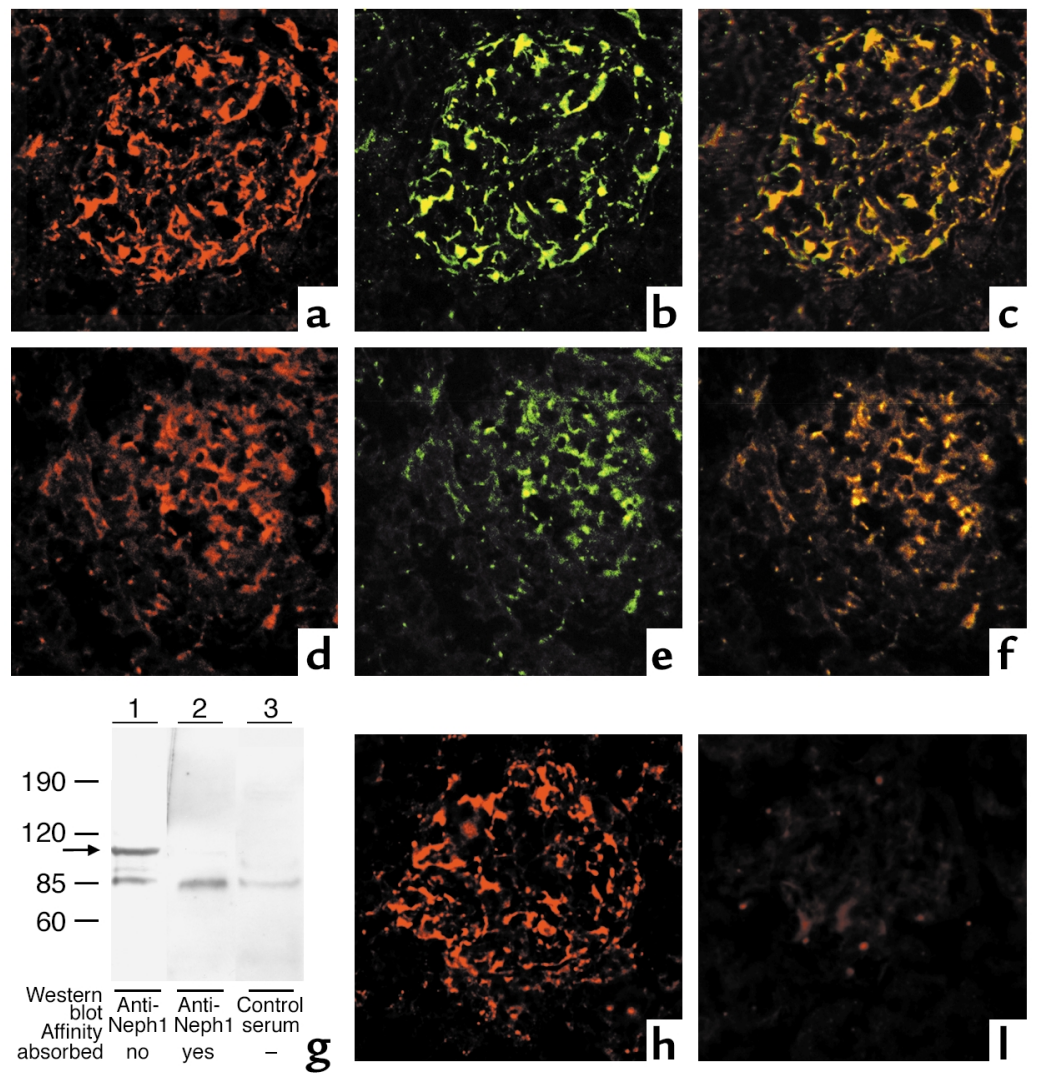

\section{Figure 3}

Localization of Neph1 in adult and developing rodent glomeruli. (a-c) Immunofluorescence images of adult rat glomeruli (3- $\mu \mathrm{m}$ sections; magnification, $\times 400)$ stained for Neph1 (a), CD2 AP (b), and merged images (c), localizing Neph 1 to podocytes. (d-f) Immunofluorescence images of day 15 developing mouse glomeruli stained for Neph1 (d), CD2 AP (e), and merged images (f), localizing Neph1 to developing podocytes. (g) Western blot of rat glomerular extracts under reducing conditions with antiNeph1 antibody prior to (lane 1) and following (lane 2) affinity absorption with a Neph1 peptide column, showing near-complete loss of immunoreactivity. (h and i) Immunofluorescence staining of $3-\mu \mathrm{m}$ rat kidney sections prior to (h) and following (i) affinity absorption of anti-Neph1 with the Neph1 peptide affinity column, showing loss of immunoreactivity.

\section{Results}

Cloning of mouse Neph1 cDNA. Cloning and sequencing by our group of the mouse cDNA fragment published by Donoviel et al. (14) revealed a single base pair disparity (absence of C after 1,204 in the Donoviel et al. sequence) which had led to a premature stop codon after 1,260 bp (Figure 1a). Combining our corresponding sequence with the 2,673-bp $3^{\prime}$ RACE product revealed a 3,255-bp clone with a stop codon 2,367 bp downstream of the open reading frame, encoding for a 789-AA protein. This sequence has been submitted to GenBank (accession number AY243095). The 2,107-bp partial rat cDNA sequence also obtained had over $95 \%$ homology at the nucleotide level and 98\% homology at the protein level with mouse Neph1 cDNA (GenBank accession number AY271309).

Structural analysis of the mouse Neph1 gene. A BLAST search of the mouse genome with our cDNA sequence revealed the presence of 15 exons spanning a 57.4-kb region of chromosome 3 , with intron 1 being disproportionately long $(42.7 \mathrm{~kb})$. Perfect identity between the predicted exons and our mouse cDNA sequence was noted. Our sequence, as well as the exons in the genomic sequence, differ from another recently submitted sequence by four base pairs (present study and Sellin et al., ref. 15) 1,064 C/T; 1,324 A/G, 2,008 A/G, 2,179 T/C).

Characterization of the mouse Neph1 protein. Domain analysis of the predicted protein sequence revealed a signal peptide (AAs 1-47), five IgG-like domains with the middle domain overlapping with a PKD-like domain, an
RGD sequence (AAs 437-439), a transmembrane domain (AAs 529-551), and a cytoplasmic tail (552-789) (Figure 1b). The most significant motifs in the cytoplasmic tail include the Grb2 SH2 site at 637 and the PDZ1 motif at 787. Another motif on the cytoplasmic tail is the Itk SH2 at 672 . However, the presence of Itk kinases in the podocyte has not yet been described, although this does not exclude potential binding by a member of the Tec kinase family at that site.

Western blot studies of native rodent glomerular protein suggest that Neph 1 exists as a monomer of about $110 \mathrm{kDa}$ under reducing conditions (Figure 2, a and b) and as a dimer and perhaps even as a multimer under nonreducing conditions (Figure 2c). These observations are confirmed by Western blot studies under nonreducing conditions with the recombinant protein that show dimeric bands of the extracellular and intracellular segments (Figure 2e), and a multimeric band of the extracellular segment. Association among Neph1 molecules is also favored by the coimmunoprecipitation of native Neph 1 by an anti-FLAG Ab from a mixture of native mouse glomerular proteins and the FLAG-tagged recombinant extracellular domain (see Figure 5b, lane 11). The $110-\mathrm{kDa}$ size of rodent Neph1 on reducing Western blot is identical to that noted by Sellin et al. (15), but differs from the $90-\mathrm{kDa}$ protein noted by Barletta et al. (27). Since the predicted molecular weight of the protein, excluding the signal peptide, is $82 \mathrm{kDa}$, slower migration on Western blot may be related to posttranslational modification of the protein. Indeed, 
there are four potential $\mathrm{N}$-glycosylation sites (at AAs 78, 172,329 , and 503), seven potential O-glycosylation sites (at AAs 56, 256, 349, 597, 698, 700, and 703), and several potential phosphorylation sites in Neph1 (Figure 1c). Indeed, deglycosylation of immunoprecipitated mouse Neph1 with PNGase F causes the protein to migrate at approximately $90 \mathrm{kDa}$ (Figure $2 \mathrm{~d}$ ).

Localization of Neph1 in adult and developing rodent glomeruli. Dual immunofluorescence staining of adult rat (Figure 3, a-c) and mouse glomeruli reveals an interrupted capillary loop pattern that is identical to, and colocalizes well with, the staining pattern for CD2 AP, suggesting that Neph1 is present in podocytes. Similar colocalization of Neph1 and CD2 AP was noted in the day 15 developing mouse kidney in the precapillary loop stage (Figure 3, d-f). In this immunofluorescence experiment, CD2 AP was used merely as a podocyte marker, since the limited resolution of immunofluorescence does not differentiate slit diaphragm proteins from other podocyte proteins present in the vicinity. Anti-Neph1 Ab depleted of immunoreactivity by absorption over a Neph 1 peptide affinity column (Figure $3 \mathrm{~g}$ ) or recombinant Neph1 extracellular segment (blots not shown) failed to stain rodent glomeruli by immunofluorescence (Figure 3, h and i). Similar results were noted with anti-nephrin absorbed with a nephrin peptide column (data not shown). Immunogold electron microscopy, which is the technique of choice for precise subcellular localization, reveals most of the 10 -nm gold particles to be present in the slit diaphragm, thereby establishing Neph1 as a slit diaphragm protein (Figure 4b). Some gold particles were also present on the foot processes in the vicinity of the slit diaphragm. The positive control for this study shows most of the 15$\mathrm{nm}$ gold particles representing CD2 AP to be present on the cytoplasmic aspect of the slit diaphragm (Figure $4 \mathrm{a})$. In a different experiment, both anti-Neph1 (Figure 4c) and anti-nephrin (Figure 4d) Ab's injected into separate rats 1 hour prior to perfusion fixation localized almost exclusively to the slit diaphragm by immunogold staining.

Overall, in a survey of ten glomeruli per study for binding of gold particles, more than $95 \%$ of the gold particles in the anti-Neph1- and anti-nephrin-injected rats were present in the slit diaphragm, compared with $75-80 \%$ in sections stained with the anti-Neph $1 \mathrm{Ab}$ in noninjected rats. The rest of the particles in both groups were equally divided between the foot process surface in close proximity to the slit diaphragm and the podocyte cytoplasm. In glomeruli stained with anti-CD2 AP, about $85 \%$ of the gold particles were present at the cytoplasmic aspect of the slit diaphragm, and the rest were present in the podocyte cytoplasm away from the slit diaphragm. Whereas Donoviel et al. had noted the presence of Neph1- $\beta$-geo fusion transcripts in podocytes, parietal epithelial cells, and mesangial cells, glomerular expression of native Neph 1 as noted by our group appears to be restricted to podocytes.
Association of Neph1 with nephrin. We conducted coimmunoprecipitation experiments to study potential interactions between three slit diaphragm proteins: Neph1, nephrin, and P-cadherin. Coimmunoprecipitation studies under nonreducing conditions using anti-Neph1 and anti-nephrin Ab's, along with the corresponding preimmune antisera, revealed that nephrin coimmunoprecipitates with anti-Neph 1 and vice versa (Figure 5a). Furthermore, we were able to coimmunoprecipitate the recombinant extracellular segment of mouse Neph1 with anti-nephrin, and vice versa (Figure $5 \mathrm{~b}$ ), but not the recombinant intracellular part (blots not shown), indicating that the Neph1-nephrin interaction occurs primarily at the level of the extracellular segments of these proteins. We were also able to demonstrate competition between FLAG-tagged and non-FLAG-tagged (unFLAG)
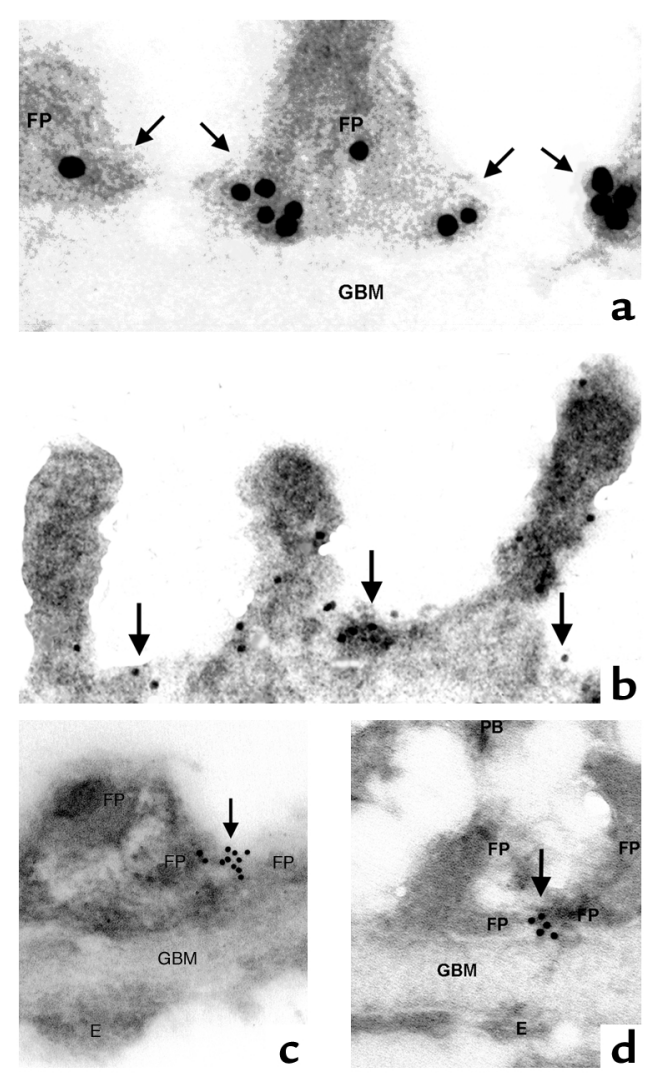

\section{Figure 4}

Immunogold localization of Neph1 to the slit diaphragm in adult rat glomeruli. (a) A control electron micrograph (magnification, $\times 50,000)$ shows staining with goat anti-CD2 AP and rabbit anti-goat IgG coupled with 15-nm gold particles localizing CD2 AP predominantly to the cytoplasmic aspect of the slit diaphragm (arrows). (b) Staining with rabbit anti-Neph1 (magnification, $\times 27,000$ ) and goat anti-rabbit IgG coupled with 10-nm gold particles, localizing Neph1 mostly to the slit diaphragm (arrows), though some labeling of the foot process is also present (see text). (c and d) Staining of kidney sections with goat anti-rabbit IgG coupled with 10 -nm gold particles (magnification, $\times 30,000$ ) from rats injected with either rabbit antiNeph1 (c) or rabbit anti-nephrin (d). Both anti-Neph1 (c) and antinephrin (d) bind specifically to the slit diaphragm (arrows), as shown by the cluster of gold particles. FP, foot process; GBM, glomerular basement membrane; E, endothelial cell; PB, podocyte body. 


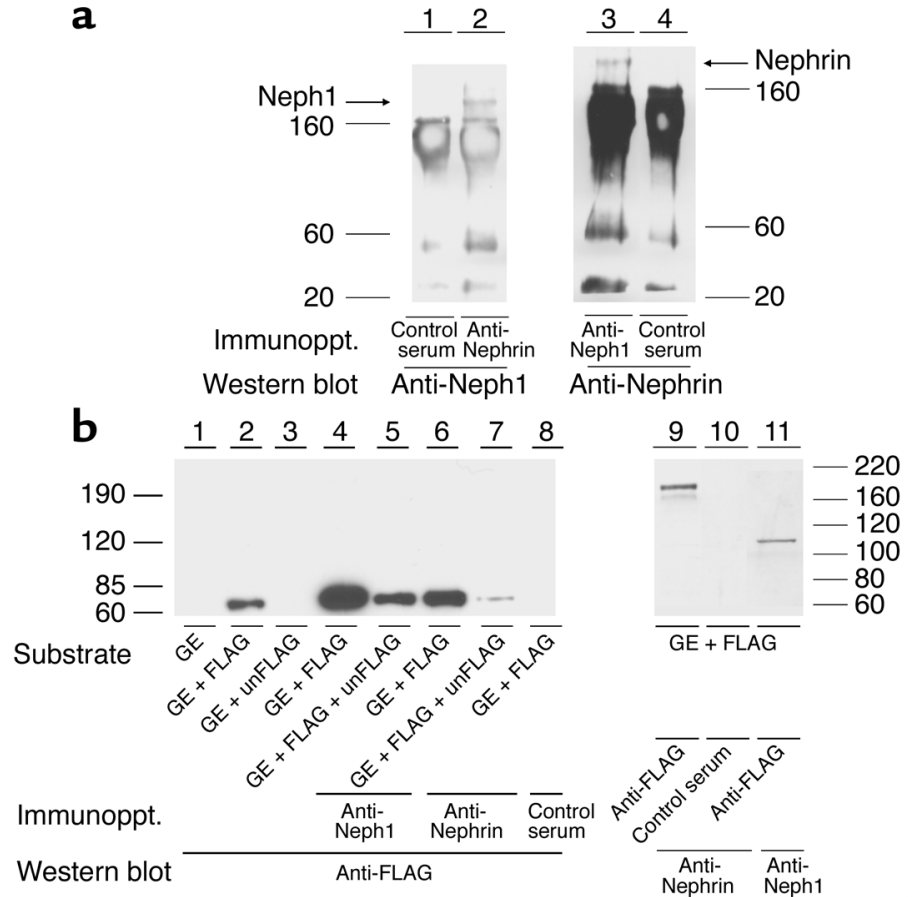

\section{Figure 5}

Composite Western blots showing the association of native (a) and recombinant (b) mouse Neph1 and nephrin. (a) Native Neph1 from mouse glomerular extracts coimmunoprecipitates with anti-nephrin (lane 2), and nephrin with anti-Neph1 (lane 3 ) under nonreducing conditions. All lanes, including control immunoprecipitations (lane 1 and lane 4), show the prominent band of nonreduced rabbit IgG at 150-160 kDa and traces of heavy chain $(50 \mathrm{kDa})$ and light chain $(25 \mathrm{kDa})$. (b) Lane 1 shows the absence of FLAG-reactive proteins in native mouse glomerular extracts. In lanes 2-11, native glomerular extracts were combined with FLAG-tagged recombinant extracellular Neph1 and also supplemented with an excess of recombinant extracellular Neph1 digested with enterokinase to remove the FLAG tag (labeled "unFLAG") in lane 5 (unFLAG/FLAG ratio, 5:1) and lane 7 (unFLAG/FLAG ratio, 10:1). Coimmunoprecipitation of recombinant Neph1 by antiNeph1 (lane 4) and anti-nephrin (lane 6); coimmunoprecipitation of nephrin (lane 9) and native Neph1 (lane 11) by antiFLAG; and competition between FLAG-tagged and non-FLAG-tagged protein (lane 5 and lane 7) are demonstrated (see text for details). GE, glomerular extract. proteins during coimmunoprecipitation studies with anti-Neph1 and anti-nephrin, as shown in Figure 5b. Lane 4 and lane 5 (unFLAG/FLAG-tagged ration, 5:1; $n=3$ readings/group) show anti-Neph1 coimmunoprecipitates; densitometry readings were 2,235 \pm 29 (lane 4) and $927 \pm 26$ (lane 5), densitometric units, $P<0.01$. Lane 6 and lane 7 (unFLAG/FLAG ratio, 10:1) show antinephrin coimmunoprecipitates; densitometry readings were $1,272 \pm 20$ (lane 6) and $49 \pm 3$ (lane 7) densitometric units, $P<0.01$ ). We were unable to coimmunoprecipitate P-cadherin with either anti-nephrin or anti-Neph1. As negative controls for the method of coimmunoprecipitation, we performed similar studies between slit diaphragm proteins (using anti-nephrin and anti-Neph1) and focal adhesion complex proteins (using anti- $\alpha$ - and anti- $\beta$-dystroglycan) or podocyte surface proteins (using anti-APA), all of which were negative (blots not shown).

In vivo study of the Neph1-nephrin association. We rationalized that disruption of slit diaphragm function would result in proteinuria and that targeting two interacting proteins critical to the barrier function would cause this disruption more efficiently than targeting them alone. To study a functional association between slit diaphragm proteins, we first injected antinephrin, anti-Neph1 (Table 1 and Figure 6a), and anti-P-cadherin Ab's (Table 2) separately into rats in increasing amounts to find a threshold nephritogenic dose that would cause mild proteinuria compared with preimmune serum injection. Dose-response curves with individual $\mathrm{Ab}$ 's helped us identify an optimum subnephritogenic dose in rats for anti-Neph1 $(300 \mu \mathrm{l}$, Figure $6 \mathrm{a}$ and Table 1$)$, anti-nephrin $(300 \mu \mathrm{l}$, Figure $6 \mathrm{a}$ and Table 1), and anti-P-cadherin (75 $\mu \mathrm{g}$, Table 2). Similarly, in mouse experiments, the optimum subnephritogenic doses were $200 \mu \mathrm{l}$ for anti-APA and $150 \mu \mathrm{l}$ for anti-nephrin and anti-Neph1. We were unable to induce any proteinuria in mice beyond baseline with anti- $\alpha$-dystroglycan and anti- $\beta$-dystroglycan. Of various subnephritogenic combinations tested, we were able to induce heterologous-phase proteinuria within the first 18 hours with anti-Neph1 and anti-nephrin; this proteinuria declined over the next 2 days (Table 3

Table 1

Urinary protein excretion in response to anti-Neph1 and anti-nephrin

Preimmune serum

Dose injected

Baseline

$100 \mu \mathrm{l}$

$300 \mu \mathrm{l}$

$500 \mu \mathrm{l}$
Anti-Neph1

$\begin{array}{cc}\text { Dose injected } & \text { Proteinuria } \\ \text { Baseline } & 0.87 \pm 0.19 \\ 100 \mu \mathrm{l} & 0.80 \pm 0.05 \\ 300 \mu \mathrm{l} & 0.80 \pm 0.40 \\ 500 \mu \mathrm{l} & 2.36 \pm 0.38^{\mathrm{A}, \mathrm{B}}\end{array}$

Anti-nephrin

$\begin{array}{cc}\text { Dose injected } & \text { Proteinuria } \\ \text { Baseline } & 0.87 \pm 0.19 \\ 100 \mu \mathrm{l} & 0.70 \pm 0.10 \\ 300 \mu \mathrm{l} & 0.80 \pm 0.09 \\ 500 \mu \mathrm{l} & 1.84 \pm 0.04^{\mathrm{A}, \mathrm{B}}\end{array}$

Protein excretion in urine over 24 hours, shown as mean \pm SE (in $\mathrm{mg}$ ), in response to injection of increasing amounts of Ab or preimmune serum into

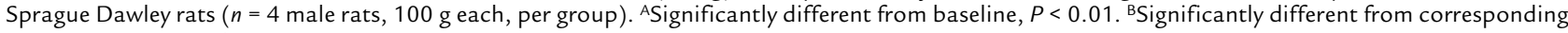
preimmune serum value, $P<0.05$. 


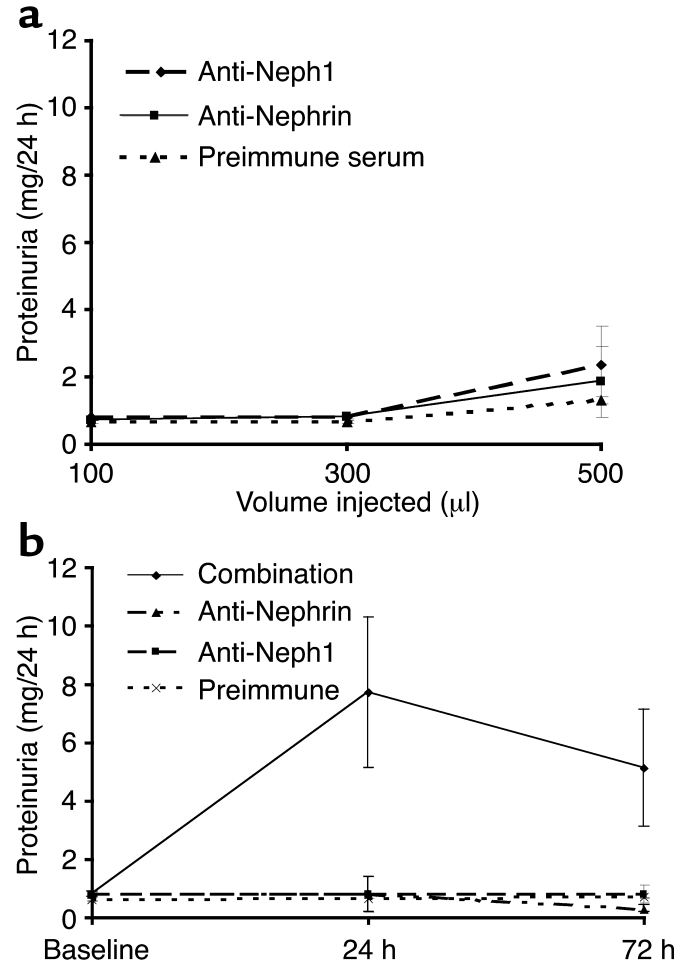

Figure 6

The induction of heterologous-phase proteinuria in rats by combinations of anti-Neph1 and anti-nephrin in individual subnephritogenic doses. (a) A dose-response plot of each individual Ab or preimmune serum to assess the optimum subnephritogenic dose. Some increase in permeability is noted at $500 \mu \mathrm{l}$ for both study Ab's compared with preimmune serum $(P<0.05)$. (b) Induction of heterologous-phase proteinuria in rats by a combination of $150 \mu \mathrm{l}$ each of anti-Neph 1 and anti-nephrin, whereas $300 \mu$ l of each individual antibody and preimmune serum do not cause proteinuria.

and Figure 6b). This experiment was repeated with older rats and revealed similar results. The proteinuria was comprised primarily of albumin, as was obvious from the approximately 70-kDa band seen on GelCode Blue-stained gels in the combination-injected group (Figure 7b); this was confirmed to be albumin by Western blot (blots not shown). The following combinations did not result in significant proteinuria beyond baseline: anti-Neph 1 and anti-P-cadherin; anti-nephrin and anti-P-cadherin; anti-nephrin and anti-APA; and anti-Neph1 and anti-APA.

Light microscopy of the kidney (hematoxylin and eosin stain) in all four groups of the anti-Neph1 and anti-nephrin experiment at 24 hours after injection showed the glomeruli to be normocellular, and staining for complement and leukocytes was negative by immunofluorescence with appropriate positive controls (images not shown). Transmission electron microscopy revealed preserved foot processes in all four groups, as exemplified by an electron micrograph from proteinuric rats at 24 hours (Figure $7 \mathrm{a}$ ). Immunofluorescence for bound rabbit IgG (anti-Neph1, anti-nephrin, or combination) revealed a change from the normal interrupted linear pattern to a cluster-like distribution in the three experimental groups starting 12 hours after intravenous injection of $\mathrm{Ab}$ (images not shown); this has been observed with several podocyte surface proteins by several investigators, including us, and appears to be unrelated to proteinuria (23). The induction of complement- and leukocyte-independent proteinuria by a combination of individually subproteinuric $\mathrm{Ab}$ doses indicates disruption of an in vivo association between nephrin and Neph1 in the slit diaphragm, without interference with foot process integrity.

As anticipated, we were unable induce proteinuria in rats using a combination of $150 \mu \mathrm{l}$ of anti-nephrin and $150 \mu \mathrm{l}$ of the Neph1 peptide column unbound fraction, reiterating the specific role played by anti-Neph 1 $\mathrm{Ab}$ 's in this process: baseline proteinuria, $0.85 \pm 0.15$ $\mathrm{mg} / 24$ hours; $\mathrm{Ab}$ injected, $0.95 \pm 0.17 \mathrm{mg} / 24$ hours (mean $\pm \mathrm{SE}, n=4$ rats weighing $100 \mathrm{~g}, P>0.05$ ). Similar results were noted when $150 \mu \mathrm{l}$ of anti-Neph1 was affinity-absorbed over a recombinant extracellular Neph 1 column and injected in combination with 150 $\mu \mathrm{l}$ of anti-nephrin: baseline, $0.85 \pm 0.15 \mathrm{mg} / 24$ hours; antibody injected, $0.92 \pm 0.15 \mathrm{mg} / 24$ hours, mean $\pm \mathrm{SE}$ $(n=4$ rats weighing $100 \mathrm{~g}$ each, $P>0.05)$. Similarly, when the anti-nephrin $\mathrm{Ab}$ used in the combination experiment was absorbed over a nephrin peptide affinity column, no significant proteinuria was induced by the combination: baseline, $0.85 \pm 0.15 \mathrm{mg} / 24$ hours; antibody injected, $0.90 \pm 0.12 \mathrm{mg} / 24$ hours $(n=4$ rats weighing $100 \mathrm{~g}$, mean $\pm \mathrm{SE}, P>0.05$ ).

Association of Neph1 with ZO-1. The presence of a PDZ-binding motif on the cytoplasmic tail of Neph1, and the absence in the literature of the direct interaction of any other slit diaphragm protein with ZO-1, prompted us to look for a potential association between Neph1 and ZO-1. As also recently shown by Huber et al. (28), we were able to coimmunoprecipitate the FLAG-tagged PDZ domains of ZO-1 with anti-Neph1, and vice versa, indicating that Neph1 and ZO-1 associate with each other on the cytoplasmic aspect of the slit diaphragm (Figure 8).

Protein and $m R N A$ expression in anti-Neph1- and antinephrin-induced slit diaphragm dysfunction. Analysis of the glomerular content of nephrin, Neph1, and ZO-1

\section{Table 2}

Urinary protein excretion in response to anti-P-cadherin

\begin{tabular}{lccc}
\hline \multicolumn{2}{c}{ Control serum } & \multicolumn{2}{c}{ Anti-P-cadherin } \\
Dose injected & Proteinuria & Dose injected & Proteinuria \\
Baseline & $1.61 \pm 0.09$ & Baseline & $1.61 \pm 0.09$ \\
$100 \mu \mathrm{l}$ & $1.56 \pm 0.05$ & $50 \mu \mathrm{g}$ & $1.60 \pm 0.60$ \\
$150 \mu \mathrm{l}$ & $1.63 \pm 0.10$ & $75 \mu \mathrm{g}$ & $1.65 \pm 0.40$ \\
$200 \mu \mathrm{l}$ & $1.66 \pm 0.03$ & $100 \mu \mathrm{g}$ & $2.40 \pm 0.05^{\mathrm{A}}$
\end{tabular}

Urinary protein excretion over 24 hours, shown as mean \pm SE (in $\mathrm{mg}$ ), in response to injection of increasing amounts of anti-P-cadherin Ab or control serum into 125 -g male Sprague Dawley rats ( $n=4$ rats/group). ASignificantly different from baseline and corresponding control serum value, $P<0.05$. 
Table 3

Urinary protein excretion in response to subnephritogenic doses of Ab's

\begin{tabular}{ccccc}
\hline & $\begin{array}{c}\text { Preimmune serum } \\
(300 \mu \mathrm{l})\end{array}$ & $\begin{array}{c}\text { Anti-Neph1 } \\
(300 \mu \mathrm{l})\end{array}$ & $\begin{array}{c}\text { Anti-nephrin } \\
(300 \mu \mathrm{l})\end{array}$ & $\begin{array}{c}\text { Anti-Neph1 }(150 \mu \mathrm{l})+ \\
\text { anti-nephrin }(150 \mu \mathrm{l})\end{array}$ \\
Day 1 & $0.65 \pm 0.02$ & $0.80 \pm 0.60$ & $0.80 \pm 0.10$ & $7.73 \pm 2.60^{\mathrm{A}}$ \\
Day 3 & $0.67 \pm 0.04$ & $0.90 \pm 0.30$ & $0.38 \pm 0.20$ & $5.10 \pm 2.07$
\end{tabular}

Urinary protein excretion over 24 hours in response to injection of subnephritogenic doses of Ab's alone or in combination ( $n=4$ male rats/group, $100 \mathrm{~g}$ each, baseline proteinuria $0.87 \pm 0.19$ ). Data are presented as mean $\pm \mathrm{SE}$, in $\mathrm{mg} / 24$ hours. ASignificantly different from corresponding values of other three groups, $P<0.05$.

proteins 24 hours after injection of the Ab's revealed modest reduction of nephrin and Neph1 in the combination group by densitometric analysis (Table 4 and Figure 9 , a and b). In addition to being substantially reduced in the combination group, $\mathrm{ZO}-1$ protein was also modestly reduced in the anti-Neph 1 group.

The results of real-time PCR are illustrated in Figure $9, \mathrm{c}-\mathrm{g}$, and the $\Delta \mathrm{Ct}$ values and $2^{-\Delta \Delta \mathrm{Ct}}$ values are summarized in Table 5. The $\Delta \mathrm{Ct}$ values of the combination versus individual $\mathrm{Ab}$-injected groups were not significantly different by Student $t$ test, and the $2^{-\Delta \Delta C t}$ values between the same groups were also less than 2 , indicating that altered gene expression did not account for the pathogenesis of proteinuria.

\section{Discussion}

Over two decades of research has provided interesting insights into the structure and function of the glomerular capillary loop (29-31). More recent studies have addressed the relative role of podocyte versus basement membrane proteins in maintaining the permeability barrier (24). The paper on Neph1 by Donoviel et al. (32) describing nephrotic syndrome at birth in Neph1 mutant mice provides conclusive evidence that Neph1 plays a significant role in the normal development and function of the glomerular capillary loop. The paper by Sellin et al. (15) describes the interaction of Neph1 with podocin in vitro, the distribution of Neph1 in a glomerular capillary loop pattern, and the transactivation of AP-1 in the presence of Tec kinases. Our current study provides conclusive evidence that Neph1 is a slit diaphragm protein, as shown by immunogold localization in the slit diaphragm.

The coimmunoprecipitation studies using antiNeph1, anti-nephrin, and anti-FLAG Ab's with glomerular extracts and recombinant proteins were highly suggestive of an interaction between Neph1 and nephrin at the level of their slit diaphragm spanning extracellular segments. We therefore decided to disrupt this interaction using an in vivo approach. The occurrence of complement- and leukocyte-independent proteinuria by a combination of anti-Neph 1 and antinephrin Ab's in individually subnephritogenic doses is highly suggestive that this interaction exists in vivo, and is at least partially altered by this Ab combination approach. Moreover, the induction of heterologousphase proteinuria by the combination of anti-Neph1 and anti-nephrin was not associated with foot process effacement or difference in the mRNA expression of ZO-1, nephrin, and Neph1 between proteinuric and nonproteinuric subgroups, indicating that it largely affected slit diaphragm permeability due to altered protein-protein interaction.

It is important to note that the doseresponse curves performed as a prelude to these studies were intended to identify an optimum subnephritogenic dose of individual $\mathrm{Ab}$ 's, and not to study the full extent of the nephritogenic potential of the individual Ab's. Indeed, both anti-Neph1 and antinephrin start increasing slit diaphragm permeability individually (compared with preimmune serum controls) at a dose of $500 \mu \mathrm{l}$ in rats. It is possible that future experiments directed toward investigating the nephritogenic potential of these individual Ab's may show more proteinuria at higher doses.

The choice of an in vivo approach to test the Neph1nephrin association over an in vitro approach using monolayers of cultured glomerular epithelial cells was made for several reasons. The slit diaphragm is the final barrier that restricts passage of plasma proteins into the urine (29). We have now known for several years that

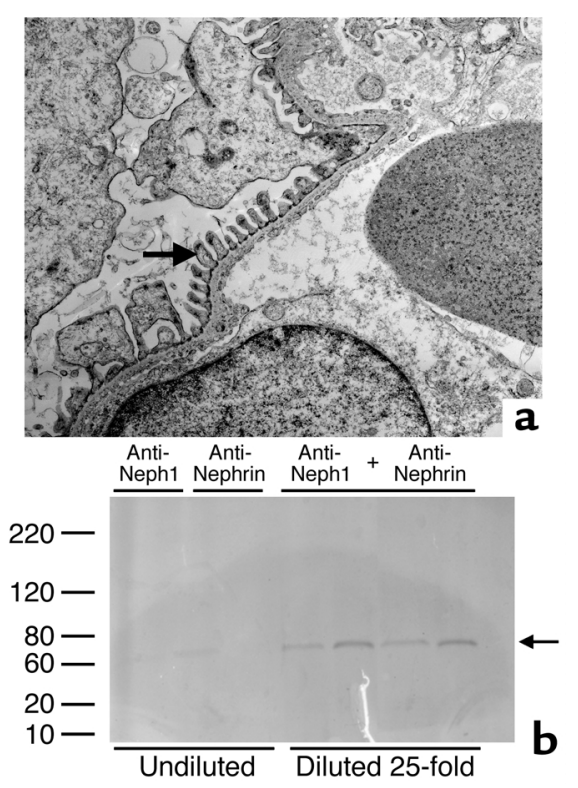

\section{Figure 7}

(a) Electron micrograph of rat kidney taken 24 hours after injection of the anti-Neph1 and anti-nephrin combination, showing that heterologous-phase proteinuria occurs in the absence of foot process effacement (arrow). Magnification, ×5,000. (b) GelCode Blue-stained reducing SDS PAGE for urinary protein from rats injected with either anti-Neph1 or anti-nephrin (undiluted), or a combination of both (diluted 25-fold). Urine from only two of four rats injected with each individual antibody is shown. The rats in the combination group showed a prominent band at approximately $70 \mathrm{kDa}$ (arrow) that was confirmed to be rat albumin by Western blot (see text). 


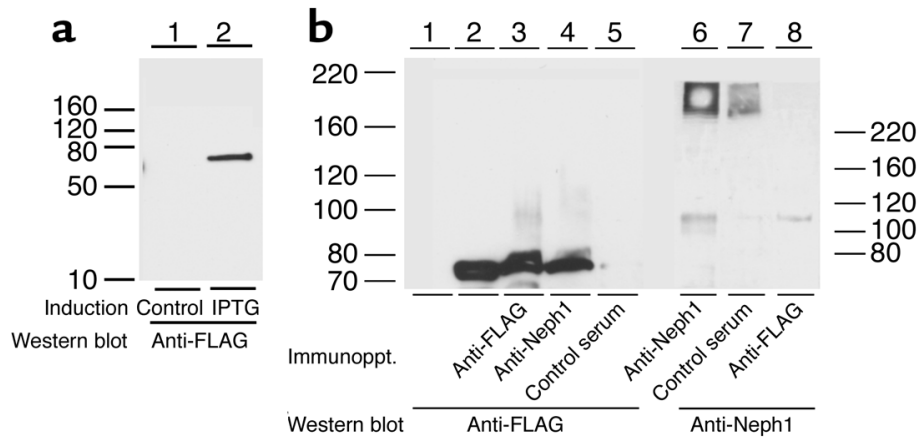

\section{Figure 8}

Composite Western blot analysis of (a) the expression of recombinant FLAG-tagged PDZ domains of mouse ZO-1 and (b) the association of Neph1 with FLAG-tagged ZO-1 PDZ domains by coimmunoprecipitation. (b) Lane 1 shows the absence of anti-FLAG reactivity in native mouse glomerular protein extracts. All other experiments shown were conducted with a combination of native mouse glomerular proteins and recombinant ZO-1 PDZ domains. Lane 4 shows the coimmunoprecipitation of ZO-1 PDZ domains by anti-Neph 1 ; lane 8 shows the coimmunoprecipitation of Neph 1 by anti-FLAG. certain Ab's directed against podocyte proteins injected intravenously induce complement- and leukocyte-independent proteinuria $(5,33,34)$. We therefore rationalized that Ab's directed against the extracellular segments of slit diaphragm proteins would bind their targets and induce slit diaphragm dysfunction that would be detectable as proteinuria. The classic example of this is $\mathrm{mAb} 5-1-6$, which induces proteinuria in rats in certain doses after binding to an epitope on the extracellular segment of nephrin. An in vitro approach to this issue would be less than ideal, since there are no cultured glomerular epithelial cells that form foot processes and slit diaphragms with morphological similarity to their in vivo counterparts. In addition, cultured cells tend to form tight junctions, which are not seen in differentiated podocytes and would make the interpretation of monolayer permeability data more difficult.

The association of ZO-1 with the cytoplasmic tail of Neph1, and the interaction of nephrin with Neph1 in the slit diaphragm, also offer valuable insights into changes in podocyte proteins during proteinuria. The study complements the astute observation of Kawachi et al. (24) that the induction of proteinuria with $\mathrm{mAb}$ 5-1-6, an anti-nephrin $A b$, results in reduced amounts of glomerular ZO-1 by immunostaining and Western blot. In the anti-Neph1/anti-nephrin model discussed above, glomerular ZO-1 was unchanged compared with controls when rats were injected with anti-nephrin at a dose that did not cause proteinuria. Some reduction of glomerular ZO-1 content was noted with the injection of anti-Neph1 alone, testifying further to the direct association of ZO-1 with Neph1. The most dramatic reduction, however, was observed when the Ab combination induced heterologous-phase proteinuria. This indicates that the interaction between nephrin and Neph1 helps to partially stabilize the association between Neph1 and ZO-1, and that disruption of this association results in a rapid reduction of glomerular ZO-1. This allows us to postulate that a nephrin/ Neph1/ZO-1 macromolecular complex exists in the slit diaphragm and may be altered in some forms of proteinuria. Whether our anti-nephrin $\mathrm{Ab}$ given alone in higher doses reduces glomerular ZO-1 will be a subject of future investigations designed to examine the nephritogenic phase of this $\mathrm{Ab}$. It would also be interesting to conduct similar studies with mAb 5-1-6.
A substantial amount of novel data presented in the Results section allows us to propose further refinements in the organization of Neph1 molecules in the slit diaphragm. The existence of Neph1 homodimers and multimers, the coimmunoprecipitation of nephrin with native Neph1, the selective coimmunoprecipitation of nephrin with the recombinant extracellular Neph1, and the induction of complement- and leukocyte-independent heterologous-phase proteinuria by the combination of anti-nephrin and anti-Neph1 Ab's in subnephritogenic doses all help in generating possible configurations. Neph1 homodimers/multimers may be arranged side by side in the slit diaphragm or adhere to each other from adjacent foot processes. It is also likely that Neph1 forms additional heterodimers with nephrin molecules either side by side or approaching each other from adjacent foot processes. Even though both the homodimeric association of Neph1 and a heterodimeric association with nephrin are both likely to be important in maintaining the permeability characteristics of the slit diaphragm, the occurrence of proteinuria after treatment with subnephritogenic combinations of Ab's rather than with individual Ab's would suggest that the latter may be more significant. In addition to the data presented above, the likelihood of a Neph1-nephrin interaction is also supported by the interaction in Drosophila between Hibris and Dumbfounded, two proteins with structural similarity to nephrin and Neph1, respectively, during myoblast fusion (35). Also, an association between nephrin and Neph1 has very recently also been shown by two other studies $(27,36)$.

\section{Table 4}

Densitometric analysis of protein expression

$\begin{array}{lllccc} & n & \text { Control } & \begin{array}{c}\text { Anti- } \\ \text { nephrin }\end{array} & \begin{array}{c}\text { Anti- } \\ \text { Neph1 }\end{array} & \begin{array}{c}\text { Anti-nephrin + } \\ \text { anti-Neph1 }\end{array} \\ \text { Neph1 } & 3 & 216 \pm 12 & 194 \pm 14 & 243 \pm 34 & 98 \pm 12^{\mathrm{A}} \\ \text { Nephrin } & 3 & 205 \pm 3 & 197 \pm 5 & 205 \pm 41 & 92 \pm 12^{\mathrm{B}} \\ \text { ZO-1 } & 5 & 440 \pm 40 & 436 \pm 56 & 153 \pm 36^{\mathrm{B}} & 33 \pm 22^{\mathrm{C}, \mathrm{D}}\end{array}$

Expression of Neph1, nephrin, and ZO-1 in rat glomeruli extracted from experimental and control groups (mean \pm SE densitometric units). ASignificantly different from corresponding control, anti-nephrin, and anti-Neph1 groups, $P<0.01$. BSignificantly different from corresponding control and anti-nephrin groups, $P<0.01$. CSignificantly different from corresponding anti-Neph1 group, $P<0.05$. DSignificantly different from corresponding control and antinephrin groups, $P<0.001$ 

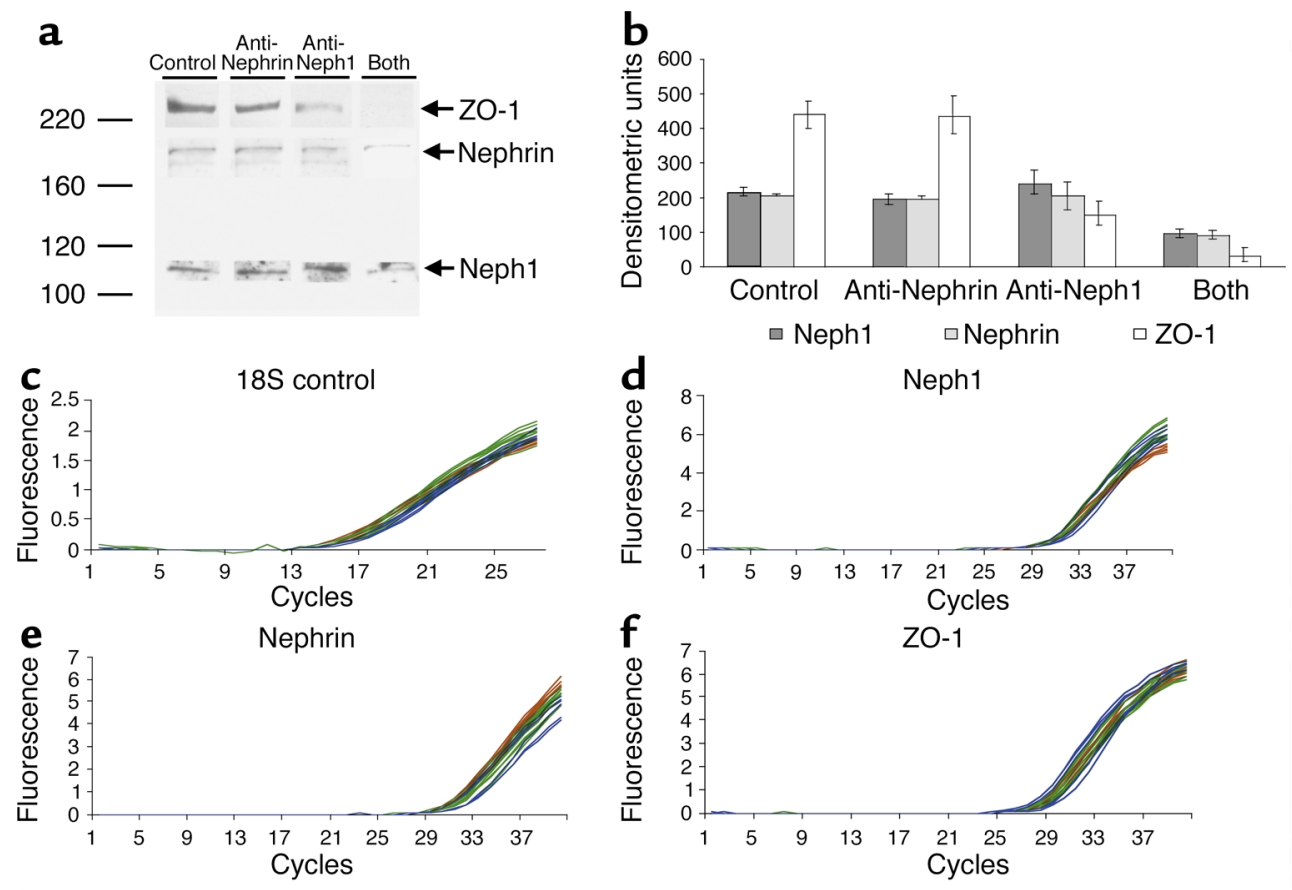

g

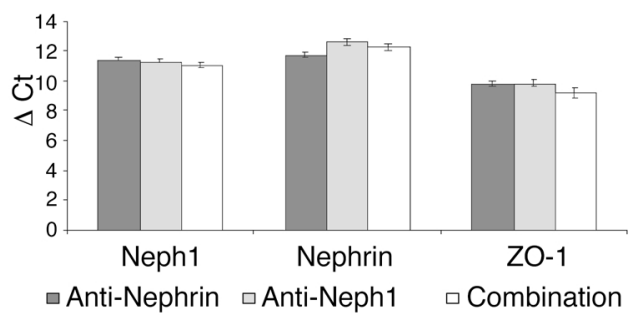

\section{Figure 9}

Composite image showing protein (a and $\mathbf{b})$ and mRNA (c-g) expression in rat glomeruli of Neph1, nephrin, and ZO-1 24 hours after the injection into rats of anti-Neph1 or anti-nephrin alone, or a combination of both. (a) Composite Western blot of glomerular extracts for each protein in the three experimental subgroups or controls. (b) Densitometric analysis of bands shown in a (mean $\pm \mathrm{SE}$ ). Modest reduction of Neph1 and nephrin and dramatic reduction of ZO-1 protein expression were noted in the combination group. Modest reduction in ZO-1 expression was also noted in the anti-Neph1 group. (c-f) Real-time PCR tracings ( $n=9$ per group) for the three genes or $18 \mathrm{~S}$ controls in the anti-nephrin (red), anti-Neph1 (green), and combination (blue) groups. (g) $\Delta \mathrm{Ct}$ (mean $\pm \mathrm{SE}$ ) values of each gene in the three groups. No significant difference in mRNA expression was noted for the individual genes between the combination and single-Ab groups $(P>0.05)$.

We have not as yet investigated the potential interactions of Neph1 or nephrin with FAT, a member of the cadherin superfamily that also spans the slit diaphragm (11). Interestingly, FAT also has a PDZ-binding motif that was not shown to bind $\mathrm{ZO}-1$ in a recent paper also addressing the association between Neph1 and ZO-1 (28). Moreover, the data appears to suggest that very large cadherins, such as the FAT family members, may not be involved in adhesion at all, but may have a sensing role (37). Also, two genes related to Neph1, named Neph2 and Neph3, have now been shown to be expressed in human kidney cortex by RT PCR (15). Precise cellular and subcellular localization is still pending. Further studies are required to investigate other protein-protein associations in the slit diaphragm that may also be important in maintaining the permeability status of this important structure.

Table 5

$\Delta C t$ values for mRNA expression of individual groups and $2^{-\Delta \Delta C t}$ values of difference between combination and individual groups by real-time $P C R$

\begin{tabular}{lccccc}
\hline & Anti-nephrin $\Delta \mathrm{Ct}$ & Anti-Neph1 $\Delta \mathrm{Ct}$ & Combination $\Delta \mathrm{Ct}$ & $\begin{array}{c}\text { Combination minus } \\
\text { anti-nephrin 2 }\end{array}$ & $\begin{array}{c}\text { Combination minus } \\
\text { anti-Neph1 2- }\end{array}$ \\
Neph1 & $11.46 \pm 0.12$ & $11.34 \pm 0.13$ & $11.07 \pm 0.15$ & $1.42 \pm 0.24$ & $1.22 \pm 0.09$ \\
Nephrin & $11.74 \pm 0.18$ & $12.62 \pm 0.19$ & $12.27 \pm 0.22$ & $0.83 \pm 0.25^{\mathrm{A}}$ & $1.13 \pm 0.05$ \\
ZO-1 & $9.85 \pm 0.19$ & $9.86 \pm 0.20$ & $9.20 \pm 0.30$ & $1.99 \pm 0.59$ & $1.67 \pm 0.24$ \\
\hline
\end{tabular}

Data are presented as mean $\pm \mathrm{SE}, n=9$ per measurement. ${ }^{A}$ Anti-nephrin minus combination was $1.60 \pm 0.45 . P>0.05$ for $\Delta \mathrm{Ct}$ between all combination and corresponding individual Ab groups. 


\section{Acknowledgments}

This work is supported by NIH grants DK-61275 to S.S. Chugh, DK-28492 and DK-60635 to Y.S. Kanwar, and the Carl W. Gottschalk Award of the American Society of Nephrology to S.S. Chugh. We thank Richard Quigg for providing rat kidney specimens injected with sheep anti-rat Fx1A and David Salant for providing sheep anti-rat nephrotoxic serum.

1. Quaggin, S.E., et al. 1999. The basic-helix-loop-helix protein pod1 is critically important for kidney and lung organogenesis. Development. 126:5771-5783.

2. Rohr, C., et al. 2002. The LIM-homeodomain transcription factor Lmx1b plays a crucial role in podocytes. J. Clin. Invest. 109:1073-1082. doi:10.1172/JCI200213961.

3. Miner, J.H., et al. 2002. Transcriptional induction of slit diaphragm genes by $\mathrm{Lmx} 1 \mathrm{~b}$ is required in podocyte differentiation. J. Clin. Invest. 109:1065-1072. doi:10.1172/JCI200213954.

4. Schnabel, E., Anderson, J.M., and Farquhar, M.G. 1990. The tight junction protein ZO-1 is concentrated along slit diaphragms of the glomerular epithelium. J. Cell. Biol. 111:1255-1263.

5. Orikasa, M., Matsui, K., Oite, T., and Shimizu, F. 1988. Massive proteinuria induced in rats by a single intravenous injection of a monoclonal antibody. J. Immunol. 141:807-814.

6. Kestila, M., et al. 1998. Positionally cloned gene for a normal glomerular protein-nephrin is mutated in congenital nephrotic syndrome. Mol. Cell. 1:575-582.

7. Shih, N.Y., et al. 1999. Congenital nephrotic syndrome in mice lacking CD2-associated protein. Science. 286:312-315.

8. Boute, N., et al. 2000. NPHS2, encoding the glomerular protein podocin, is mutated in autosomal recessive steroid-resistant nephrotic syndrome. Nat. Genet. 24:349-354.

9. Kaplan, J.M., et al. 2000. Mutations in ACTN4, encoding alphaactinin-4, cause familial focal segmental glomerulosclerosis. Nat. Genet. 24:251-256.

10. Reiser, J., Kriz, W., Kretzler, M., and Mundel, P. 2000. The glomerular slit diaphragm is a modified adherens junction. J. Am. Soc. Nephrol. 11:1-8.

11. Inoue, T., et al. 2001. FAT is a component of glomerular slit diaphragms. Kidney Int. 59:1003-1012.

12. Ihalmo, P., Palmen, T., Ahola, H., Valtonen, E., and Holthofer, H. 2003. Filtrin is a novel member of nephrin-like proteins. Biochem. Biophys. Res. Commun. 300:364-370.

13. Topham, P.S., et al. 1999. Nephritogenic monoclonal antibody 5-1-6 is directed against the extracellular domain of rat nephrin. J. Clin. Invest. 104:1559-1566.

14. Donoviel, D.B., et al. 2001. Proteinuria and perinatal lethality in mice lacking NEPH1, a novel protein with homology to NEPHRIN. Mol. Cell. Biol. 21:4829-4836.

15. Sellin, L., et al. 2003. NEPH1 defines a novel family of podocin interacting proteins. FASEB J. 17:115-117.
16. Mundel, P., and Shankland, S.J. 2002. Podocyte biology and response to injury. J. Am. Soc. Nephrol. 13:3005-3015

17. BLAST. www.ncbi.nlm.nih.gov/BLAST.

18. Scansite. http://scansite.mit.edu/.

19. ExPASy Proteomics tools. http://ca.expasy.org/tools/\#primary.

20. SignalP V1.1 World Wide Web Prediction Server. www.cbs.dtu.dk/services/SignalP

21. DAS - Transmembrane Prediction Server. www.sbc.su.se/ miklos/DAS/

22. ExPASy ProtScale. http://ca.expasy.org/cgi-bin/protscale.pl.

23. Chugh, S., et al. 2001. Aminopeptidase A: a nephritogenic target antigen of nephrotoxic serum. Kidney Int. 59:601-613.

24. Kawachi, H., et al. 1997. Slit diaphragm-reactive nephritogenic MAb 5-1-6 alters expression of ZO-1 in rat podocytes. Am. J. Physiol. 273:F984-F993.

25. Wada, J., and Kanwar, Y.S. 1998. Characterization of mammalian translocase of inner mitochondrial membrane (Tim44) isolated from diabetic newborn mouse kidney. Proc. Natl. Acad. Sci. U. S. A. 95:144-149.

26. Kanwar, Y.S., et al. 1996. D-glucose-induced dysmorphogenesis of embryonic kidney. J. Clin. Invest. 98:2478-2488.

27. Barletta, G.M., Kovari, J.A., Verma, R.K., Kerjaschki, D., and Holzman, L.B. 2003. Nephrin and Neph1 co-localize at the podocyte foot process intercellular junction and form cis hetero-oligomers. J. Biol. Chem. 278:19266-19271.

28. Huber, T.B., et al. 2003. The carboxyl terminus of neph family members binds to the PDZ domain protein zonula occludens-1. J. Biol. Chem. 278:13417-13421.

29. Kanwar, Y.S., and Venkatachalam, M.A. 1992. Ultrastructure of the glomerulus and juxta-glomerular apparatus. In Handbook of renal physiology. E.E. Windhager, editor. Oxford University Press. New York, New York, USA. 3-40.

30. Wieslander, J., et al. 1984. Goodpasture antigen of the glomerular basement membrane: localization to noncollagenous regions of type IV collagen. Proc. Natl. Acad. Sci. U. S. A. 81:3838-3842.

31. Abrahamson, D.R., and Caulfield, J.P. 1982. Proteinuria and structural alterations in rat glomerular basement membranes induced by intravenously injected anti-laminin immunoglobulin G. J. Exp. Med. 156:128-145

32. Hamano, Y., et al. 2002. Determinants of vascular permeability in the kidney glomerulus. J. Biol. Chem. 277:31154-31162.

33. Assmann, K.J.M., van Son, J.P.H.F., Dijkman, H.B.P.M., and Koene, R.A.P. 1992. A nephritogenic rat monoclonal antibody to mouse aminopeptidase A. J. Exp. Med. 175:623-635.

34. Mentzel, S., van Son, J.P., Dijkman, H.B., Wetzels, J.F., and Assmann, K.J. 1999. Induction of albuminuria in mice: synergistic effect of two monoclonal antibodies directed to different domains of aminopeptidase A. Kidney Int. 55:1335-1347.

35. Dworak, H.A., Charles, M.A., Pellerano, L.B., and Sink, H. 2001. Characterization of Drosophila hibris, a gene related to human nephrin. Development. 128:4265-4276.

36. Gerke, P., Huber, T.B., Sellin, L., Benzing, T., and Walz, G. 2003. Homodimerization and heterodimerization of the glomerular podocyte proteins nephrin and NEPH1. J. Am. Soc. Nephrol. 14:918-926.

37. Angst, B.D., Marcozzi, C., and Magee, A.I. 2001. The cadherin superfamily. J. Cell Sci. 114:625-626. 